\title{
Advanced Network Management Systems: A Risk-Based AC OPF Approach
}

\author{
Sahban W. Alnaser, Graduate Student Member, IEEE, and Luis F. Ochoa, Senior Member, IEEE
}

\begin{abstract}
This paper presents a risk-based advanced distribution Network Management System (NMS) aimed at maximizing wind energy harvesting whilst simultaneously managing congestion and voltages. The NMS allows the adoption of multi-minute control cycles so the volume of actions from onload tap changers (OLTCs) and distributed generation (DG) plants can be reduced whilst effectively catering for the effects of wind power uncertainties. This work presents the quantification of benefits and impacts from adopting different control cycles as well a comparison with a deterministic approach. The risk-based approach is implemented by adapting and expanding an AC Optimal Power Flow. A risk level is used to determine the extent to which congestion and voltage rise could exist during a control cycle. The proposed NMS is applied to a real-life UK MV network from the North West of England to assess its effectiveness in managing high penetration of wind power considering minute by minute simulations for one week. The results show that the risk-based approach can effectively manage network constraints better than the deterministic approach, particularly for multi-minute control cycles, reducing also the number of control actions but at the expense of higher levels of curtailment.
\end{abstract}

Index Terms-- Active network management, distributed generation, generation curtailment, optimal power flow, uncertainty.

\section{INTRODUCTION}

$\mathrm{T}$ HE transition towards a low carbon society is placing considerable operational challenges for Distribution Network Operators (DNOs) to accommodate the increasing levels of renewable Distributed Generation (DG). DNOs traditionally resolve voltage constraint and congestion issues at the planning stage by reinforcing the existing network assets or limiting the installed DG capacity, i.e., providing a firm connection to new DG plants (free to operate up to their rated output). This "Fit \& Forget" approach, however, may increase the required cost and connection time which in turn represent a barrier to connect more DG capacity [1]. This is even more important in the context of wind energy, due to their inherent variability and consequent low usage of assets.

An active approach to facilitate the deployment of larger volumes of renewable DG with cheaper and quick connection is the application of curtailment, where set points are defined

This work was supported in part by the University of Jordan, Jordan.

The authors are with the Electrical Energy and Power Systems (EEPS) Group, School of Electrical and Electronic Engineering, The University of Manchester, Manchester M13 9PL, UK.

(email: sahban.alnaser@postgrad.manchester.ac.uk, luis_ochoa@ieee.org) for DG plants in order to manage network constraints, such as congestion [2]. In those cases, the real power outputs of DG plants are actively controlled based on pre-determined set of rules to decrease the exports through a particular corridor of the distribution network. Such schemes, however, require Smart Grid-like infrastructure to increase network observability and controllability.

As more DG is connected it is likely that more Active Network Management (ANM) approaches that also cater for other network elements (such as on-load tap changes[3-4] and storage [5]) will be needed in order not to only resort to DG curtailment. Such a scenario where multiple DG plants and network elements could be actively managed for the benefit of the network but also minimizing curtailment requires more complex control approaches than rule-based solutions. This is a paradigm shift towards an intelligent, centralized, advanced Network Management System (NMS) [6].

The design of an advanced NMS must handle the uncertainties introduced by wind power (i.e., variability) and avoid/reduce the risk to violate network constraints [7-9]. Indeed, 'optimal' decisions taken for a given control cycle might not be adequate for the next one, leading to binding constraints or poor wind energy harvesting.

Advanced or future NMS algorithms have been proposed in the literature in the context of high penetrations of wind power in distribution networks. A decision making algorithm is proposed in [10] to find the most adequate set points based on a predefined search space generated through simulations or based on historical performance, to mange voltage constraints using OLTCs. In [11], real-time measurements are considered using the Constraint Satisfaction Problem (CSP) method to obtain the best DG set points (i.e., curtailment) to solve congestion issues. However, the algorithms in [10-11] may fail to cope with more complex networks that include significant number of control devices. In [12], an Optimal Power Flow (OPF) approach is applied to minimize DG curtailment but without including OLTCs. Moreover, all the aforementioned NMS approaches do not provide a complete, integrated solution for both congestion and voltage problems. An integrated approach is considered in [13-14]. However, the optimization problem is formulated in a simplified manner using linear programming and voltage sensitivity coefficients.

From the control perspective, the above studies, except from [10-12], do not consider the system response between successive control actions, making it impossible to adequately model the real-time operation and, thus, evaluate the performance of the controller. Furthermore, all previous 
studies do not consider firm generation in the modeling of NMS (all DG units are controllable), which is necessary when investigating cases with existing connection agreements (the uncontrollable power output increases the challenge to manage network constraints). Although in [11-12] and [15] multisecond control cycles are used to quickly react to wind variations, the above decision-making algorithms do not explicitly formulate the uncertainty of wind power.

To tackle the aforementioned challenges not yet been adequately addressed in the literature, this work proposes a comprehensive risk-based NMS algorithm aimed at managing in pseudo real-time network simultaneously voltages and congestion through intelligent, coordinated control of OLTCs, DG power factor, and DG curtailment (as last resort). The proposed risk-based NMS allows investigating in detail the benefits and impacts of adopting different control cycles, quantifying in particular compliance of voltages with the EN50160 standard [16], congestion levels, capacity factor of wind farms and, crucially, volume of control actions. The AC OPF (i.e., a non-linear programming problem) proposed in [17] is adapted and expanded to produce a risk-based AC OPF that caters for wind power uncertainties, particularly during multi-minute control cycles to reduce the volume of control actions. Optimal set points for the active elements are obtained to both minimize DG curtailment and reduce/avoid operational problems.

Here, the proposed NMS is applied to a real-life UK MV network from the North West of England considering firm and controllable DG. The performance of this risk-based NMS is evaluated through a time-series analysis for one week using one-minute resolution data for both demand and generation and considering different control cycles (1, 5 and 15 minutes).

This paper is structured as follows: Section II presents the NMS architecture and overview of the operational aspects. The formulation of the optimization engine is explained in section III. The methodology to handle the uncertainty due to wind power is presented in section IV. In sections V and VI, the results from the application of the proposed NMS on a real-life MV network with multiple wind farms are presented and discussed. Practical implementation aspects are discussed in section VII. Conclusions are drawn in section VIII.

\section{NMS ARCHITECTURE AND OPERATION: OVERVIEW}

The proposed NMS platform is shown in Fig. 1. Real-time measurements (in practice managed by a SCADA system) are obtained from time-series simulations using OpenDSS [18]. Network elements are monitored in order to identify branches (i.e., lines or transformers) exceeding their thermal capacities or nodes with voltages outside the statutory limits. Here, the time interval between two consecutive constraint checks is defined as "control cycle". The AC OPF-based optimization engine, implemented in the modeling language AIMMS [19], finds the best set points for the available controllable resources (i.e., OLTCs, DG power factor, and DG curtailment) upon the violation of constraints or the existence of curtailment. In practice, given the need for accurate information, the SCADA system should interface with a more intelligent way of

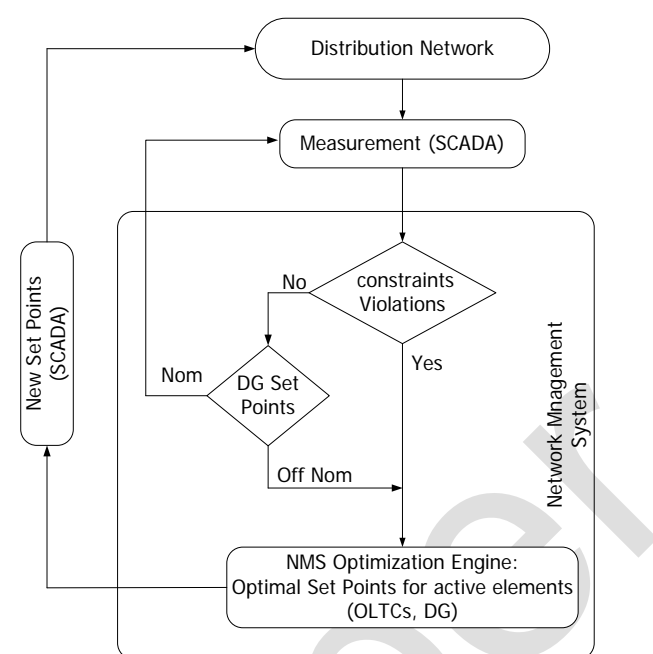

Fig. 1 Architecture of the proposed NMS.

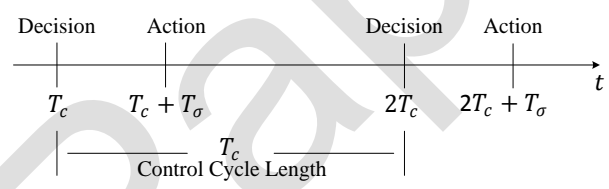

Fig. 2 Control cycle of the proposed NMS.

processing monitoring data and extending network observability, i.e., a state estimator. However, this is out of scope of this work and it is assumed that the measurements are perfectly received without errors, correctly time stamped, and the network is fully observed.

From the operational perspective, Fig. 2 shows the details of the adopted parameters for the control cycle. At the start of each control cycle, $T_{c}$, the NMS observes the measurements in the network. When constraints are violated, a decision is made to produce the optimum set points for the active elements. These set points are then applied (action) after a delay of $T_{\sigma}$ (that in practice could appear due to processing time or communications). These set points will be held throughout the length of control cycle $T_{c}$.

The above is considered in this work as a deterministic approach. Within this approach set points are identified to solve only the issues at the start of the control cycle. These set points may not be suitable for the entire cycle (e.g., due to wind gusts) and hence may lead to the violation of constraints. To cater for this, it is necessary the deployment of predictive decision- making algorithms in the NMS that account for the possible changes in the network conditions (i.e., due to wind power) within the control cycle. The proposed optimization engine extends the deterministic approach by considering optimal set points that solve not only the network issues seen at the start of the control cycle but throughout it - hence catering for the stochastic nature of wind. This facilitates the adoption of multi-minute control cycles (instead of multisecond) allowing a significant reduction in the volume of the required control actions.

The intensity and the probabilities of changes of wind power outputs are obtained by analyzing historical data. This is used to find the $K$ likely scenarios of wind power changes (more details in section IV). Once the corresponding scenario 
tree is established, it will be considered by the optimization engine as $K$ simultaneous AC OPF problems linked by probabilistic and non-probabilistic constraints. Considering all the scenarios at once makes it possible to find the set points that satisfy all of them. This approach is known as ChanceConstrained Programming [20] which is an approach within Stochastic Programming.

The non-probabilistic constraints aim to maintain voltages and power flows within limits to eliminate the issues that triggered the controller. The probabilistic constraints will use the probabilities of the $K$ scenarios to 'weigh' the voltage excess or thermal overloads for a given element. This overall value -that caters for all scenarios leading to issues- is then compared with a predefined risk level. This risk level will allow going 'beyond' the non-probabilistic constraints. In practice, the most adequate risk level will only be defined after assessing the performance of the proposed NMS for different values as this depends on the particular characteristics of the network and the wind resource.

\section{NMS OPTIMIZATION ENGINE}

At the start of each control cycle, the state of loads, firm/controllable generators and network elements (i.e., tap positions of OLTCs) for a given instant are sent from OpenDSS (in practice, the SCADA system) to the proposed AC OPF-based NMS optimization engine. To run the AC $\mathrm{OPF}$, in addition to the states above, an identical network representation is considered (i.e., topology, impedances, nominal capacity of loads and generators, etc.).

The proposed AC OPF formulation, as a proxy to minimizing DG curtailment, maximizes the total active power that could be injected by the controllable wind farms (set $N$ indexed by $n$ ), as given below.

$$
\sum_{n \in N} S P_{n}\left(\frac{p_{n}}{S P_{n}^{0}}\right)
$$

where $S P_{n}$ represents the new set point for the corresponding control cycle, $p_{n}$ is the active power output and $S P_{n}^{0}$ the original set point for the last control cycle.

With this objective function, the OPF will find the most adequate set point (2) that harvests as much as possible the available wind resource (active power injection is divided by the set point used throughout the last control cycle). In practice, the available wind resource can be obtained by either directly measuring the wind power output or indirectly using wind speed measurements and the power characteristics of the wind turbines.

$$
0 \leq S P_{n} \leq 1 \quad \forall n \in N
$$

The optimization will be carried out in a framework that considers the available information at the decision time and the $K$ scenarios (indexed by $k$ ) throughout the control cycle. Each scenario, obtained from a statistical analysis of historical wind data, has a probability and a magnitude of the potential wind power change (more details in section IV). These $K$ changes (relative to nominal capacity) are denoted by $\omega_{g, k}$ and $\omega_{n, k}$, for firm generation (set $G$ indexed by $g$ ) and controllable
DG plants, respectively, and with a probability $\operatorname{Pr}(k)$.

The following subsections present the general AC OPF constraints (such as active/reactive power balance), the nonprobabilistic constraints for the $k=0$ scenario, and finally the probabilistic constraints for all the scenarios.

\section{A. General AC OPF Constraints}

Active $f_{l, k}^{(1,2),(P)}$ and reactive power $f_{l, k}^{(1,2),(Q)}$ injections at the start and end buses (denoted 1 and 2) for each line and each transformer (all represented by the set $L$ indexed by $l$ ) are calculated according to the standard KVL expressions for active, $f_{l, k, K V L}^{(1,2),(P)}(V, \delta)$, and reactive power, $f_{l, k, K V L}^{(1,2),(Q)}(V, \delta)$, as presented below.

$$
\begin{array}{cc}
f_{l, k}^{(1,2),(P)}=f_{l, k, K V L}^{(1,2),(P)}(\boldsymbol{V}, \boldsymbol{\delta}) & \forall l \in L, \forall k \in K \\
f_{l, k}^{(1,2),(Q)}=f_{l, k, K V L}^{(1,2),(Q)}(\boldsymbol{V}, \boldsymbol{\delta}) & l \in L, \forall k \in K
\end{array}
$$

The distribution network is supplied by at least one point interfacing with the upstream grid. It is assumed that this point $x$ ( $X$, set of external connections) can import/export real and reactive power $\left(p_{x, k}, q_{x, k}\right)$ within limits $\left(p_{x}^{(-,+)}, q_{x}^{(-,+)}\right)$. One of these external connections is taken as the slack bus with voltage angle, $\delta_{\text {slack, },}$, equal to zero.

If the transformers are equipped with OLTCs, the corresponding terms in (3) and (4) for voltage at the start bus of the line will be divided by the tap ratio $\tau_{l}$. The OLTCs are controlled using min and max tap positions $M^{(-,+)}$to achieve voltage regulation capabilities in the range $\left[\tau_{l}^{-}, \tau_{l}^{+}\right]$. To avoid tap changes beyond the actual operational capabilities of the OLTCs (considering one minute resolution) and to reduce the effects of wearing, the tap change is limited to one (up or down). The corresponding regulation equivalent $R_{l}$ (in per unit, e.g., 0.0167) is used for this.

$$
\left|\tau_{l}-\tau_{l}^{0}\right| \leq R_{l}
$$

In this work, the (almost) real-time operation of locally controlled OLTCs is also modeled. To cater for this within the optimization, the values of the tap positions are considered as fixed parameters.

Given that the AC OPF is formulated as a non-linear programming problem (i.e., continuous variables), the tap ratio requires a post-optimization adjustment. It should be converted to the nearest discrete value that reflects a feasible integer tap position $m_{1} \in\left\{M^{-}, \ldots,-1,0,1, \ldots, M^{+}\right\}$.

$$
m_{l}=\text { nearest int }\left(\frac{\tau_{l}-1}{R_{l}}\right)
$$

The firm generation is operating at fixed power factor angle $\emptyset_{g}$, while the controllable DG units can operate at different power factors $\emptyset_{n}$. This makes reactive power "dispatchable" within the MVA capability of the corresponding DG plant $\emptyset_{n}^{(-,+)}$. The formulation of this capability can also adopt the corresponding P-Q curve [21].

KCL describes the balance of real and reactive power at each bus (set $B$ indexed $b$ ), and for each scenario $k$, according to (7) and (8), respectively. Here, the potential wind power changes, $\omega_{g, k}$ and $\omega_{n, k}$, are incorporated in the balance 
equations by multiplying the corresponding nominal capacities of the DG plants ( $p_{g}^{\text {rated }}$ and $p_{n}^{\text {rated }}$ ). These wind power changes are applied to both firm and controllable DG plants.

$$
\begin{gathered}
\sum_{n \in N \mid \beta_{n=b}} S P_{n}\left(\left(\frac{p_{n}}{S P_{n}^{0}}\right)+\omega_{n, k} p_{n}^{\text {rated }}\right) \\
+\sum_{g \in G \mid \beta_{g=b}}\left(p_{g}+\omega_{g, k} p_{g}^{\text {rated }}\right) \\
+\sum_{x \in X \mid \beta_{x=b}} p_{x, k}=d_{b}^{P}+\sum_{l \in L \mid \beta_{l}^{(1,2)}=b} f_{l, k}^{(1,2)(P)} \\
\sum_{n \in N \mid \beta_{n=b}} S P_{n}\left(\left(\frac{p_{n}}{S P_{n}^{0}}\right)+\omega_{n, k} p_{n}^{\text {rated }}\right) \tan \left(\emptyset_{n}\right)+ \\
\sum_{g \in G \mid \beta_{g=b}}\left(p_{g}+\omega_{g, k} p_{g}^{\text {rated }}\right) \tan \left(\emptyset_{g}\right)+ \\
\sum_{x \in X \mid \beta_{x=b}} q_{x, k}=d_{b}^{Q}+\sum_{l \in L \mid \beta_{l}^{(1,2)}=b} f_{l, k}^{(1,2),(Q)}
\end{gathered}
$$

where $\beta_{u}$ maps the location of each network element ( $u \subset$ $\{n, g, x, l\})$ to its associated bus, and $d_{b}^{(P, Q)}$ are the active and reactive demand at the same bus.

\section{B. Non-Probabilistic Constraints (Scenario $k=0$ )}

Even if wind power changes are likely throughout the control cycle, it is crucial to ensure that the voltage and/or congestion issues seen at the start of the cycle are effectively eliminated. To implement this, the corresponding wind power output (scenario $k=0$ ) has to be considered in a deterministic fashion (as discussed in section II).

Thus, the voltage magnitude at bus $b\left(V_{b}\right)$ should be within the statutory limits $V_{b}^{(-,+)}$and the apparent start/end power flows through lines and transformers should be limited to their corresponding thermal capacity $f_{l}^{+}$.

$$
\begin{gathered}
V_{b}^{-} \leq V_{b, k=0} \leq V_{b}^{+} \quad \forall b \in B \\
\left(f_{l, k=0}^{(1,2), P}\right)^{2}+\left(f_{l, k=0}^{(1,2), Q}\right)^{2} \leq\left(f_{l}^{+}\right)^{2} \quad \forall l \in L
\end{gathered}
$$

\section{Probabilistic Constraints (all scenarios)}

While it is crucial to solve the network issues seen at the start of the control cycle, it is also paramount to incorporate the effects of the potential changes in wind power output (during the control cycle). Consequently, in addition to the previous constraints, probabilistic inequality constraints are incorporated to tackle the uncertainty brought by wind power.

With the proposed probabilistic constraints, the OPF allows voltages to rise above the statutory limit and power flows through lines or transformers are also allowed to exceed their thermal capacities. However, the intensity of voltage excursions and congestions are controlled using a pre-defined risk level $\varepsilon$ as given in (11) for voltage excursions and in (12) for thermal violations:

$$
\sum_{k \in K} \Delta_{b, k}^{V} \operatorname{Pr}(k) \leq \varepsilon \quad \forall b \in B
$$

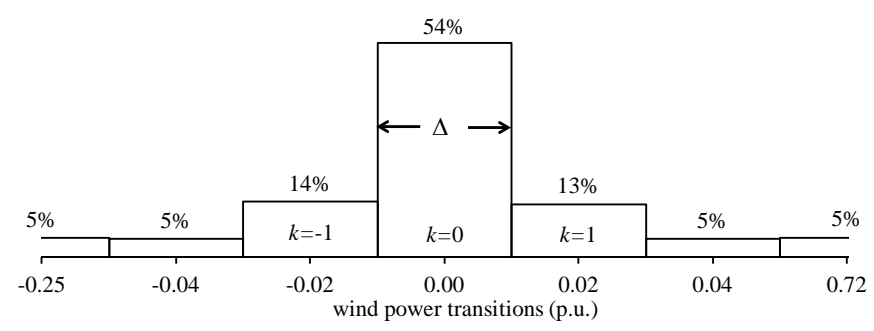

Fig. 3 Probability distribution of 1-min wind power data using 5-min period.

$$
\sum_{k \in K} \Delta_{l, k}^{S} \operatorname{Pr}(k) \leq \varepsilon \quad \forall l \in L
$$

Where $\Delta_{b, k}^{V}$ is voltage excursion at bus $b$ and $\Delta_{l, k}^{S}$ is the percentage of thermal violation for branch $l$, where its calculation is given in (13).

$$
\Delta_{l, k}^{S}=\left\{\begin{array}{c}
0, \quad f_{l, k}^{(1,2)} \leq f_{l}^{+} \\
\frac{f_{l, k}^{(1,2)}-f_{l}^{+}}{f_{l}^{+}}, \quad f_{l, k}^{(1,2)}>f_{l}^{+}
\end{array}\right\} \forall l \in L
$$

In addition, due to BS EN50160 standard [16] adopted in the UK, voltages can never exceed $10 \%$ of nominal.

$$
V_{b, k} \leq 1.1 \quad \forall b \in B
$$

Moreover, to avoid congestion beyond emergency limits, a further constraint is also included (15).

$$
\left(f_{l, k}^{(1,2), P}\right)^{2}+\left(f_{l, k}^{(1,2), Q}\right)^{2} \leq\left(f_{l}^{\text {emergency }}\right)^{2} \forall l \in L
$$

Large values of $\varepsilon$ (close or equal to 1 ) mean that constraints (11) and (12) will accept all type of voltage and congestion violations. This will make the non-probabilistic constraints (9) and (10) become the dominant ones, forcing the set points to only be optimized for scenario $k=0$. Therefore, irrespective of the adopted risk levels $(\varepsilon)$, the risk-based approach can perform at worst as the deterministic approach. The extent to which this performance can be improved will depend on the selection of the risk level, as well as the characteristics of the network, demand and wind resource.

\section{Production of the SCEnARio TreE}

Because of the short time horizon needed by the proposed NMS (control cycles from 1 to 15 minutes), it is assumed that the availability of the wind resource remains constant (also known as persistence forecasting) [22]. However, the potential changes or errors, called here transitions, need to be computed so the corresponding uncertainties are taken account of.

This section presents the methodology to produce the scenario tree of wind power changes and the corresponding probabilities and magnitudes. For this, one-minute resolution historical normalized wind power data is statistically analyzed using different periods such as 1,5 and 15 minutes. These periods will correspond to the control cycles to be adopted by the NMS optimization engine.

First, the one-minute resolution wind power profile is segmented by the length of the defined period. The magnitudes (changes in normalized wind power) of the intrasegment transitions are then calculated. For instance, for a period of 5 minutes, segments will have 5 transitions: min 0 to $\min 1$, min 0 to $\min 2$, and so on. The corresponding magnitudes of these intra-segment transitions are then stored 


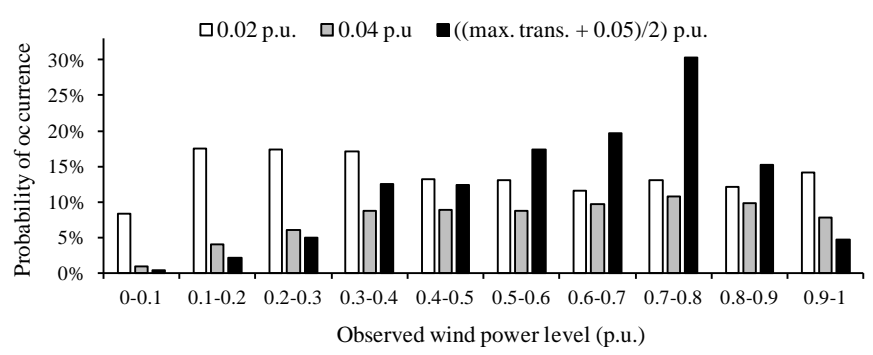

Fig. 4 Probability density function for the positive wind power transitions using 5-min period.

in a pool for the whole historical wind power profile.

The number of scenarios $K$ has to be defined. This number, however, has to be a trade-off between a better representation of potential wind power changes (more scenarios) and the corresponding computational burden. It also has to be an odd number in order to consider the scenario $\mathrm{k}=0$.

The distribution produced by the pool of transitions is divided into $K$ 'bins' as shown in Fig. 3. The mean value of each bin will correspond to the magnitude of the scenario. The size of each bin $(\Delta)$ has to be carefully determined to reduce errors due to approximation. Adopting large values leads to more transitions inside a single bin (e.g., scenario $k=0$ ), hiding actual changes.

In addition, the production of scenarios (intensity and probability) has to be done according to the wind power output to better represent the potential changes. For instance, the potential changes for wind power below 0.1 p.u. might not be the same as for when the power injections are as high as 0.8 p.u. To tackle this issue, the 'binning' process is done considering different subsets of the pool of transitions. Each subset will contain only those transitions that depart from a given range of wind power output (e.g., 0.8p.u.-0.9p.u.).

To illustrate the process, the normalized wind power profile for a site in England is analyzed. This data corresponds to February 2010 and has one-minute resolution. The pool of transitions considers a control cycle of 5 minutes and 10 different subsets for wind power output ranges at the start of each defined period [0-0.1], (0.1-0.2], ..., (0.9-1] p.u. In this work, seven scenarios are considered: one without changes $(k=0)$, three with positive changes $(k=1,2,3)$ and three with negative changes $(k=-1,-2,-3)$. Due to the characteristics of the historical data, $\Delta$ was considered as 0.02 p.u. (or $2 \%$ of wind power change relative to nominal capacity). Thus, the bins are (-min. trans., -0.05$),[-0.05,-0.03),[-0.03,-0.01),[-0.01$, $0.01),[0.01,0.03),[0.03,0.05)$, and $[0.05$, max. trans). The minimum and maximum transition values will depend on the corresponding subset. The corresponding magnitudes for the scenarios are $-($ min. trans. +0.05$) / 2,-0.04,-0.02,0,0.02$, 0.04 , and (max. trans. +0.05 ) $/ 2$.

The resulting probability density function (pdf) for the positive transitions per wind power range at the start of the control cycle is shown in Fig. 4. This pdf (similar to the negative transitions) clearly highlights the importance of the differentiation per wind power range. Indeed, as expected, when the wind does not blow the likely changes are smaller than when there is more resource.

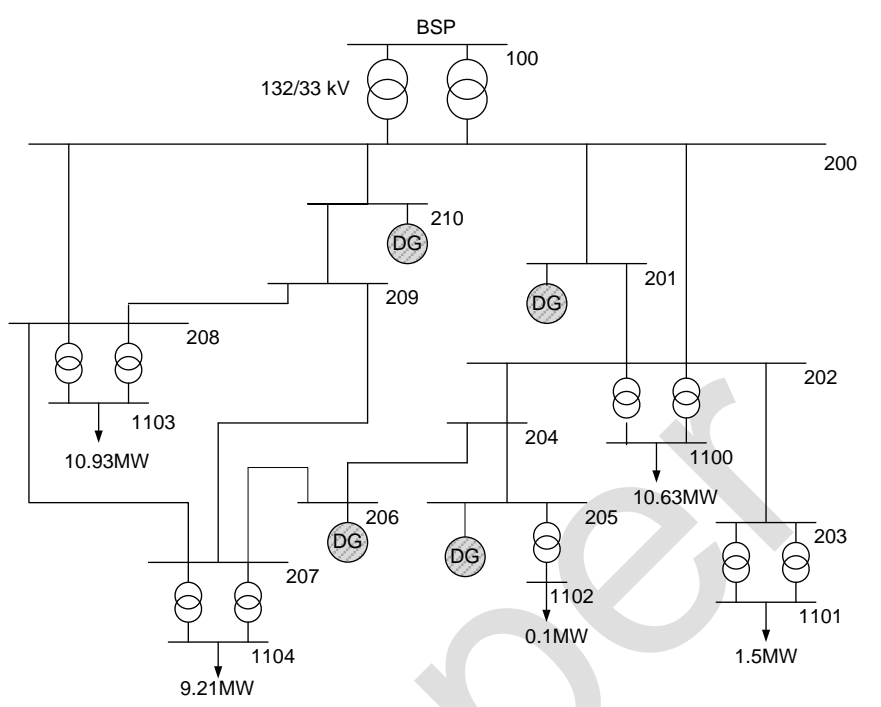

Fig. 5 Real-life UK MV network from the North West of England.

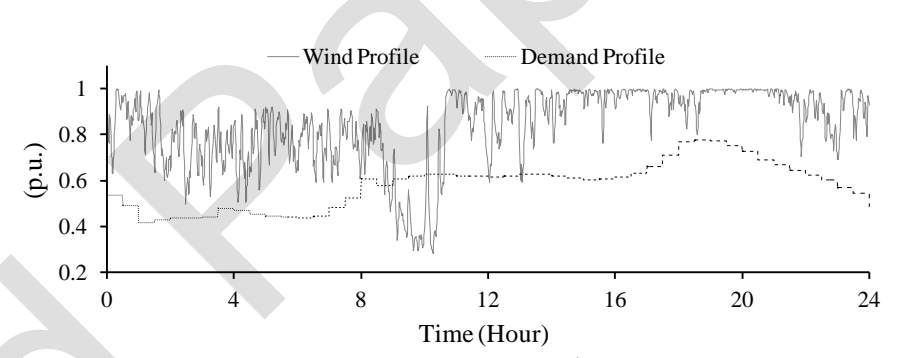

Fig. 6 Normalised wind and demand profiles on $1^{\text {st }}$ February 2010.

\section{CASE Study: ReAL-Life MV NETWORK}

The proposed NMS is applied to the real-life UK MV network from the North West of England. The single line representation of the network is given in Fig. 5. The $33 \mathrm{kV}-$ feeders are supplied by two 63 MVA 132/33 kV transformers at the Bulk Supply Point (BSP). The OLTCs at the BSP control the secondary voltage to 1.00 p.u. and has a range of $+10 \%$ to $-20 \%$ in 18 steps of $1.67 \%$ and a voltage deadband of $\pm 3 \%$. The primary substations $33 / 11 \mathrm{kV}$ are equipped with OLTCs using a pre-defined voltage target of 1.0 p.u. at the busbar and a deadband of $\pm 1.5 \%$ (aligned with UK practices). The regulation range on the primary sides of $-17.16 \%$ to $+5.72 \%$ and tap steps of $1.43 \%$. The network initially has four DG plants with firm connection agreements at buses 201, 205, 206 and 210 with capacities of 9.1, 10.6, 12 and 7.5MW, respectively.

The maximum and the minimum demands for the whole network are 31 and $12 \mathrm{MW}$, respectively. Half-hourly load profiles at each primary substation are based on the corresponding number and type of customers. Demand and 1min wind profiles are shown in Fig. 6 for $1^{\text {st }}$ February 2010.

To demonstrate the real-time operation of the proposed NMS controller in managing congestions and maintaining voltage within statutory limits ( $\pm 6 \%$ of nominal), controllable DG is connected at buses 205 and 206 with capacities of 20 and $15 \mathrm{MW}$, respectively, that create significant congestion at line 204-205 (thermal capacity of 23 MVA) and voltage rise at bus 205. The new controllable DG units are operating with unity power factor. To illustrate the proposed risk-based NMS 

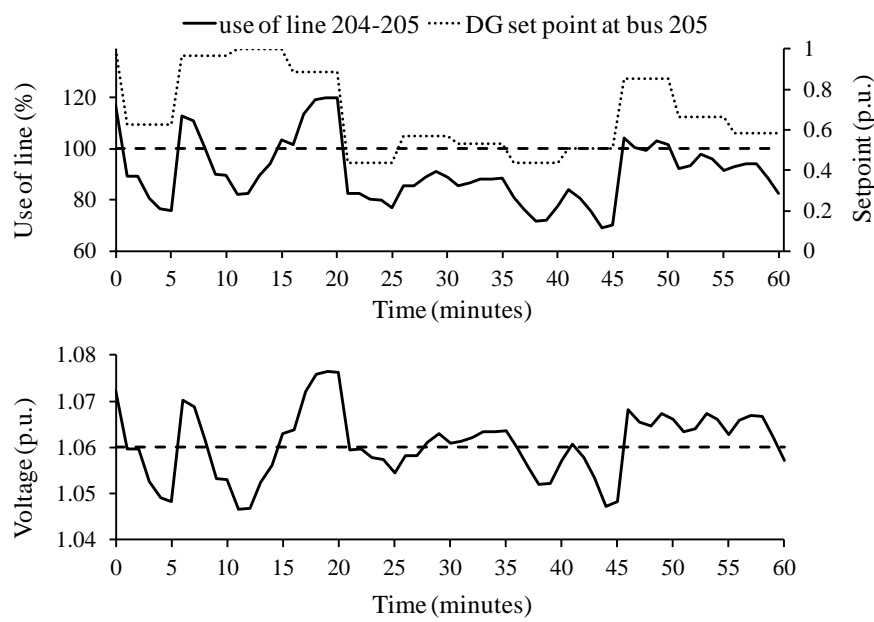

Fig. 7 Deterministic curtailment-only NMS using 5-min control cycle: (top) use of line 204-205 p.u. and set point for the wind farm at bus 205 p.u.; and (bottom) voltage at bus 205p.u..
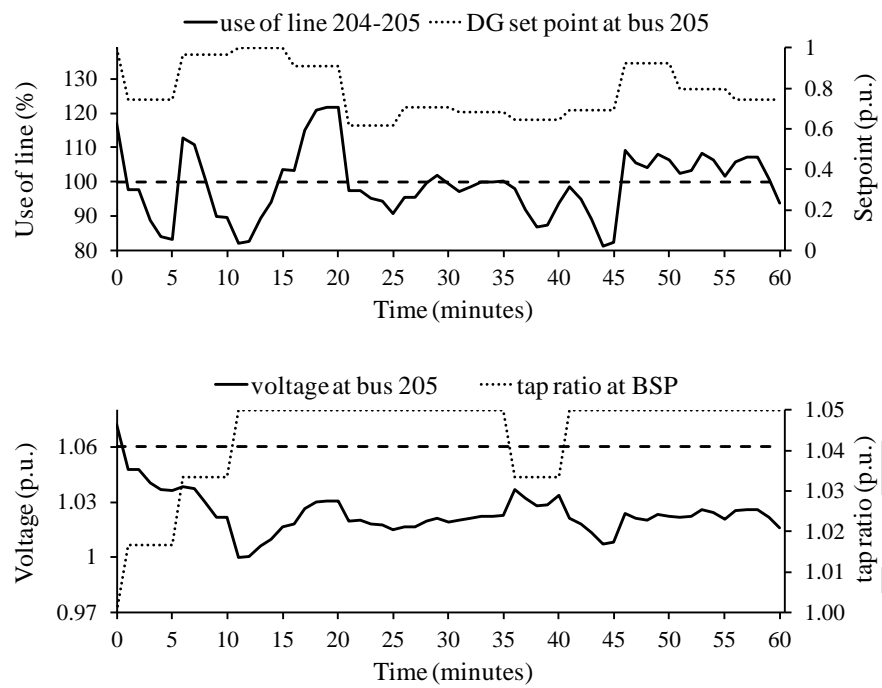

Fig. 8 Deterministic curtailment and CVC NMS using 5-min control cycle: (top) use of line 204-205 p.u. and set point for the wind farm at bus 205 p.u.; and (bottom) voltage at bus 205 p.u. and tap ratio of the OLTC.

approach, first the simpler deterministic NMS (optimal decision based on measurements at the start of the control cycle) will be presented. The analyzed period is 60 minutes with a 5-min control cycle (sufficient to assess the multiminute control cycle performance).

The set points produced by the AC OPF, for both deterministic and risk-based approaches, are applied simultaneously but considering a response of one minute to mimic the corresponding mechanical and communication delays of the controllable devices. In practice, due to different time responses and potential hunting effects, a staged approach should also be investigated.

\section{A. Deterministic Approach}

In the deterministic approach the optimal set points are identified to solve network issues seen at the start of the control cycle but are adopted throughout the same cycle. To avoid potential issues (such as wind gusts), multi-second control cycles can be adopted (as shown in [11-12] and [15]). However, this very short control cycles would increase the
TABLE I

Wind Power Scenarios at minute 15

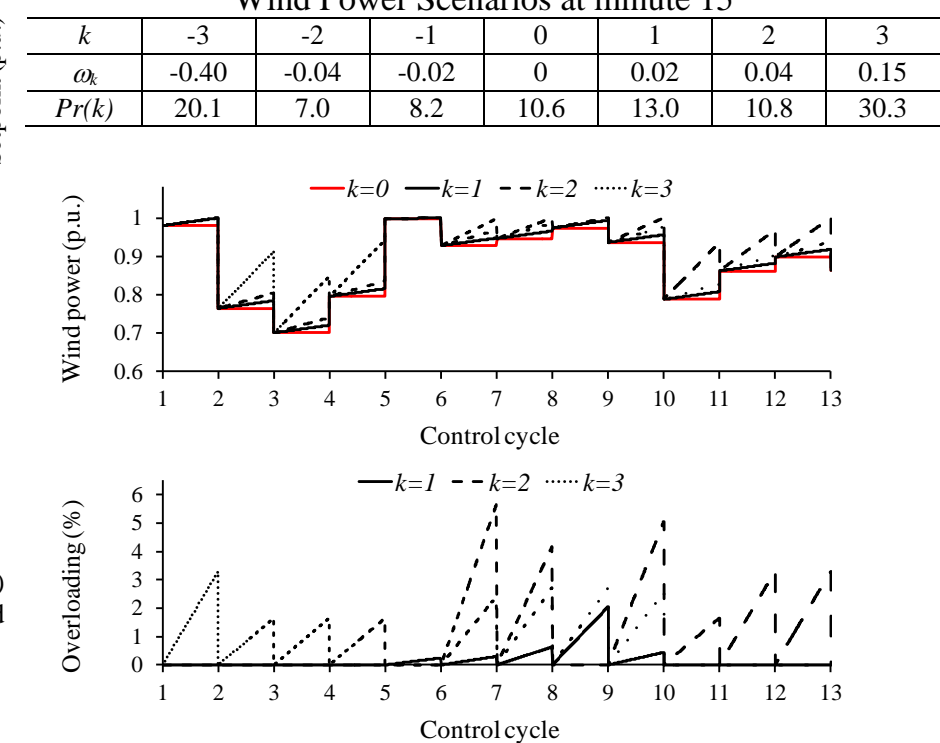

Fig. 9 (Top) Scenarios of wind power changes and the corresponding (bottom) thermal overloading for line 204-205 (\%) for curtailment and CVC NMS using a 5-min control cycle.

volume of the control actions, accelerating the aging of elements such as OLTCs or even the wind turbines (due to pitch angle control). In addition, delays due to the mechanical response of elements as well as communication delays limit the practical length of the control cycle. Based on this, the shortest control cycle considered in this work is 1 minute.

First, a deterministic curtailment-only NMS scheme is applied. Fig. 7 shows the one-minute resolution loading of line 204-205, the voltage profile at bus 205, and the set point of the corresponding wind farm. The other controllable DG unit (206) is not curtailed by the NMS during this period.

At the start of the simulation, the controller is activated to simultaneously solve congestion (excess of $17 \%$ ) and voltage rise (1.07 p.u.). Based on the optimization engine, the NMS actions the wind farm at bus 205 to change its set point $\left(S P_{D G_{-} 205}\right)$ from the initial $100 \%$ (1.00p.u.) to 63\%. This setting is kept through the control cycle. At minute 5 (next control cycle), there is no constraint violation. However, given that curtailment has been applied to the wind farm, the NMS triggers the optimization to find a new set point that increases the harvesting. The set point is improved at minute 6 to $97 \%$.

To explore the benefits from additional flexibility, Coordinated Voltage Control (CVC) is applied to the OLTC at the BSP. As it can be seen in Fig. 8, during the first three control cycles the tap ratio increases (i.e., secondary voltage decreases) to minimize curtailment whilst maintaining the voltage at bus 205 within the statutory limits. In addition, when voltage is the main issues, the DG set point tends to be much higher compared to the curtailment-only scheme, e.g., at minute 30 , the set point is improved from 53\% to $68 \%$.

Although the deterministic approach does improve network performance, the voltage rise issues are not managed effectively in the curtailment-only scheme and congestion 
TABLE II

Deterministic and Risk-Based NMS

\begin{tabular}{|c|c|c|c|c|c|c|c|c|c|}
\hline \multirow[b]{2}{*}{$\begin{array}{l}\text { Control } \\
\text { Cycle }\end{array}$} & \multirow[b]{2}{*}{ NMS Scheme } & \multirow{2}{*}{\multicolumn{2}{|c|}{ NMS Approach }} & \multicolumn{6}{|c|}{$\begin{array}{rr}\text { Performance metrics } \\
\end{array}$} \\
\hline & & & & $\begin{array}{c}\text { Total Length of } \\
\text { Overloads for } \\
\text { 200-201 (min) } \\
\end{array}$ & $\begin{array}{l}\text { Longest Overload } \\
>15 \% \text { for } 200-201 \\
\text { (min, avg overload) } \\
\end{array}$ & $\begin{array}{c}\text { EN50160 } \\
\text { Compliance of } \\
\text { Bus } 205(\%) \\
\end{array}$ & $\begin{array}{l}\text { BSP Tap } \\
\text { Changes }\end{array}$ & $\begin{array}{l}\text { TV for } \\
\text { all DG }\end{array}$ & $\begin{array}{c}\text { Curtailed } \\
\text { Energy (\%) }\end{array}$ \\
\hline \multirow{8}{*}{ 5-min } & \multirow{4}{*}{$\begin{array}{l}\text { Curtailment } \\
\text { Only }\end{array}$} & \multicolumn{2}{|c|}{ Deterministic } & $892 \mathrm{~min}$ & $4 \min , 20 \%$ & 89 (no) & - & 39 & 14.4 \\
\hline & & \multirow{3}{*}{$\begin{array}{c}\text { Risk- } \\
\text { Based }(\varepsilon)\end{array}$} & 0.005 & $471 \mathrm{~min}$ & 2 min, $19 \%$ & 90 (no) & - & 32 & 17.0 \\
\hline & & & 0.001 & $145 \mathrm{~min}$ & $2 \min , 16 \%$ & 94 (no) & - & 29 & 18.7 \\
\hline & & & 0 & $72 \mathrm{~min}$ & $2 \mathrm{~min}, 16 \%$ & 99 (yes) & - & 26 & 20.5 \\
\hline & \multirow{4}{*}{$\begin{array}{c}\text { Curtailment + } \\
\text { CVC + } \\
\text { PF control }\end{array}$} & \multicolumn{2}{|c|}{ Deterministic } & $903 \mathrm{~min}$ & $4 \mathrm{~min}, 23 \%$ & 96 (yes) & 89 & 32 & 10.1 \\
\hline & & \multirow{3}{*}{$\begin{array}{c}\text { Risk- } \\
\text { Based }(\varepsilon)\end{array}$} & 0.005 & $444 \min$ & 2 min, $18 \%$ & 96 (yes) & 59 & 26 & 13.3 \\
\hline & & & 0.001 & $143 \mathrm{~min}$ & $2 \mathrm{~min}, 16 \%$ & 96 (yes) & 43 & 24 & 14.6 \\
\hline & & & 0 & $76 \mathrm{~min}$ & $2 \mathrm{~min}, 16 \%$ & 98 (yes) & 33 & 23 & 15.3 \\
\hline \multirow{4}{*}{ 1-min } & \multirow{4}{*}{$\begin{array}{c}\text { Curtailment + } \\
\text { CVC + } \\
\text { PF control }\end{array}$} & \multicolumn{2}{|c|}{ Deterministic } & 727 min & $1 \mathrm{~min}, 19 \%$ & 96 (yes) & 305 & 68 & 10.2 \\
\hline & & \multirow{3}{*}{$\begin{array}{c}\text { Risk- } \\
\text { Based }(\varepsilon)\end{array}$} & 0.005 & $383 \mathrm{~min}$ & none & 97 (yes) & 300 & 66 & 11.8 \\
\hline & & & 0.001 & $88 \mathrm{~min}$ & none & 97 (yes) & 101 & 65 & 13.4 \\
\hline & & & 0 & $28 \mathrm{~min}$ & none & 98 (yes) & 69 & 57 & 14.2 \\
\hline \multirow{4}{*}{ 15-min } & \multirow{4}{*}{$\begin{array}{c}\text { Curtailment + } \\
\text { CVC + } \\
\text { PF control }\end{array}$} & \multicolumn{2}{|c|}{ Deterministic } & $938 \mathrm{~min}$ & $12 \min , 24 \%$ & 95 (yes) & 33 & 13 & 10.3 \\
\hline & & \multirow{3}{*}{$\begin{array}{c}\text { Risk- } \\
\text { Based }(\varepsilon)\end{array}$} & 0.005 & $569 \mathrm{~min}$ & $5 \mathrm{~min}, 20 \%$ & 96 (yes) & 29 & 10 & 13.5 \\
\hline & & & 0.001 & $235 \mathrm{~min}$ & $5 \mathrm{~min}, 20 \%$ & 97 (yes) & 15 & 9.5 & 14.8 \\
\hline & & & 0 & 160 min & $5 \mathrm{~min}, 20 \%$ & 98 (yes) & 15 & 9.3 & 15.4 \\
\hline
\end{tabular}
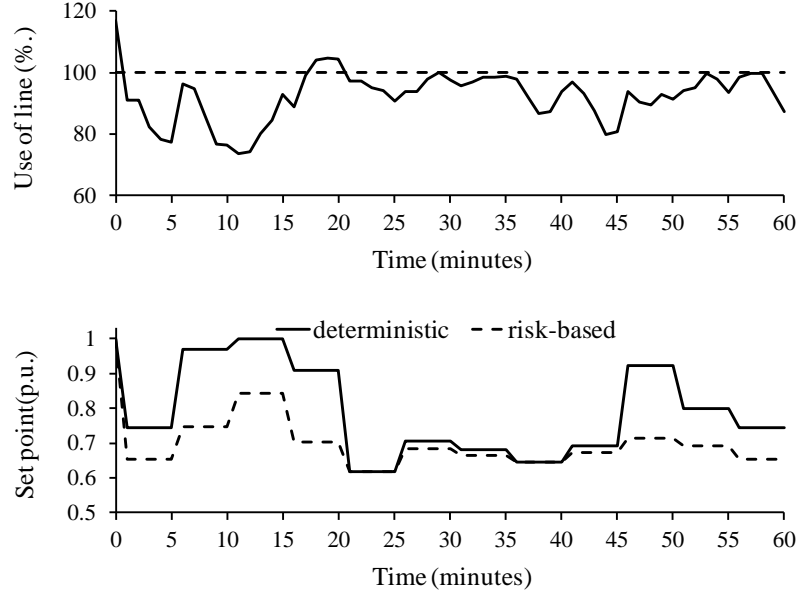

Fig. 10 Curtailment and CVC NMS using a 5-min control cycle: (top) use of line 204-205 p.u. using risk-based approach $(\varepsilon=0.005)$; and (bottom) deterministic and risk-based set points for the wind farm at bus 205 p.u.

exists for many minutes for both schemes. This is because the optimal settings solve the network issues at the start of 5-min control cycle but disregard the potential wind power changes within the cycle. For instance, in Fig. 8, the set point at minute 15 is reduced to $91 \%$ to tackle congestion. At the time of effective control action at minute 16, the wind resource increases throughout the control cycle and the line is overloaded again by $21 \%$.

The above illustrates the need to incorporate the potential wind power changes into the NMS optimization engine to hedge against undesirable scenarios that may lead to the violation of network constraints.

It is worth noting that in practice the implementation of a deterministic approach would use voltage and/or congestion operating margins to minimize the excursions throughout the control cycles [2]. This, however, would be at the expense of more curtailment and hence requires tuning. For clarity and consistency, operating margins were not taken into account in either of the control approaches, deterministic and risk-based.

\section{B. Risk-Based Approach}

In order to use the proposed risk-based NMS, first a risk level has to be adopted. This value has to be found on a caseby-case basis after performing multiple analyses. Here, for simplicity, a value of $0.5 \%(0.005)$ is adopted. As mentioned before, seven scenarios of wind power changes are considered. The intensity and probability of the seven wind power changes will be updated each control cycle based on the statistical analysis of historical data. To illustrate this, at minute 15 (start of the $4^{\text {th }}$ control cycle), all the corresponding intensity and probabilities of scenarios are shown in Table I. Based on the scenarios at the start of each control cycle the optimization engine will find the best set points to minimize curtailment whilst managing network constraints within the specified risk level $(\varepsilon)$.

Considering both curtailment and CVC, Fig. 9 shows the scenarios of wind power increases at the start of the control cycle (top) and the corresponding thermal overloading (bottom) at line 204-205 (the other scenarios do not lead to overloads). The resulting risk for a given control cycle, i.e., the sum of overloads multiplied by the corresponding probability of the scenario, must be lower than the specific risk level (as presented in (12)). For instance, for the $4^{\text {th }}$ control cycle, congestion only occurs with scenario $k=3$, exceeding the limit by $1.65 \%$. This multiplied by its probability (30.3\%) results in an overall risk below 0.005 . Note, when the wind power value is close to unity (e.g., at the $5^{\text {th }}$ control cycle), thermal overloading for all scenarios are almost eliminated.

Fig. 10 shows the usage of line 204-205 and the optimal DG settings produced by the proposed risk-based NMS. Compared to the deterministic approach, it is clear that congestion issues have been reduced dramatically in frequency (from 26 to 5 minutes) and in magnitude. For the latter, during the $4^{\text {th }}$ cycle, the overload went down from $21 \%$ to $4 \%$. This was achieved by considering that the potential wind power 
increase of 0.15 p.u. (of nominal capacity) had a $30.3 \%$ chance of occurring. As for voltages, although issues were also solved deterministically, the number of tap changes reduced slightly (two tap changes).

In this case with a 5-min control cycle, the risk-based approach also allows reducing the volume of control actions from the DG plant at bus 205. The total variation (TV) of the DG set point, defined as the sum of absolute changes in the set points [23], is reduced by a third when compared to the deterministic approach (from 1.5 to 1.0 p.u.).

In a multi-minute framework, the issue of having a more accurate approach to manage network constraints that also leads to a reduction in the volume of control actions is, however, the increase in curtailment. As shown in Fig. 10, the DG set point for the risk-based approach is in general below that of the deterministic one.

It is important to highlight that the performance of the proposed risk-based NMS depends on the specified risk level. Lower values will force the probabilistic constraints to be more restrictive of congestion and voltage rise in all scenarios. Higher values, however, will relax the probabilistic constraints potentially resulting in more congestion and voltage rise that at worst (i.e., $\varepsilon=1.0$ ) are equal to the deterministic approach.

\section{PERformance Assessment}

This section demonstrates the performance of the proposed NMS optimization engine considering the usage of different control cycle lengths, ANM schemes, and risk levels $(\varepsilon)$. Moreover, to produce a more complex environment, the network previously used will now be studied considering a significantly higher wind penetration.

The multi-period AC OPF planning tool developed in [4], which considers active schemes (but neglects real-time operation aspects), is applied to calculate the maximum additional DG capacity that could be connected to the four locations with firm DG (buses 201, 205, 206 and 210). Adopting wind and generation data for February 2010, PF control, CVC, 2\% curtailment, and a $10 \%$ congestion allowance, this planning tool suggests that an extra $53 \mathrm{MW}$ (135\% more DG capacity) could be connected (5, 17, 22 and 9 MW at buses 201, 205, 206, and 210, respectively). The connection of this capacity without control would not be in practice possible since it overloads lines 200-201, 204-205 and $200-210$ beyond $25 \%$ of their thermal capacities. In addition, the voltage at bus 205 would exceed the upper limit for more than a quarter of the time.

Similar to the previous section, one-minute resolution simulations for the first week of February 2010 are carried out applying different control solutions to this new generation portfolio (53 MW of controllable DG units). All the generators are capable of operating with power factors between 0.95 inductive and capacitive according to UK requirements [24].

Metrics are applied to assess the performance during the analyzed period. To assess the effectiveness of the approach to manage congestion, two metrics are used: total length of overloads (in minutes); and the longest duration and the corresponding average magnitude of overloads larger than
$15 \%{ }^{1}$. For voltages, the BS EN50160 [16] standard (95\% of 10 -min voltage magnitudes must be within $\pm 6 \%$ of nominal), is used to appraise the NMS performance against voltage rise problems. To evaluate the volume of control actions, two metrics are used: the number of BSP tap changes; and the total variation (TV) of the DG set points (the sum of absolute changes [23]). Finally, the curtailed energy is also computed.

Table II compares the performance of the deterministic and the risk-based approaches (with different risk levels) for the curtailment-only and the full NMS schemes (with CVC and power factor control). Three control cycle lengths are investigated: 1, 5 and 15 minutes. Results for thermal overloads and voltages are presented for line 200-201 and bus 205 , respectively, as they were the most affected.

In the deterministic approach using a 5-min control cycle, the use of the CVC and PF control schemes leads to smaller volumes of curtailed energy (10.1\%) compared to the curtailment only scheme (14.4\%). In addition, the full NMS scheme allows maintaining voltages within the statutory limits for $95.5 \%$ of the analyzed week (i.e., EN50160 compliant).

Adopting shorter control cycles allows monitoring the network status more frequently and closely following the variability of wind resource. This results in a better management of congestion and voltages. For instance, in the deterministic approach using a 1-min control cycle, the longest overload (average of $19 \%$ ) lasts for only 1 minute. These figures go up to $24 \%$ lasting for 12 minutes using a 15-min control cycle. However, this improvement is at the expense of the control actions: the number of BSP tap changes and TV are, respectively, 10 and 5 times bigger for a 1-min cycle.

The risk-based NMS always manages congestions and voltage excursions better than the deterministic approach. For instance, the curtailment-only scheme with a 5-min control cycle and $\varepsilon=0$ is EN50160 compliant and does not use the OLTC. However, the curtailment goes up to $20.5 \%$. This effective network management allows adopting multi-minute control cycles to reduce the volume of control actions. For example, in the full NMS scheme, the effectiveness of the risk-based approach to manage congestion using a 5-min control cycle and 0.005 risk-level is similar to the 1 -min control cycle of the deterministic approach. Consequently, it allows adopting a 5-min control cycle which in turn dramatically reduces the number of tap changes and the TV of DG plants by 80 and 62\%, respectively.

The risk level also has a significant influence on performance. The full NMS scheme with $\varepsilon=0.005$ manages to significantly reduce congestion issues for all control cycles when compared to the deterministic approach. Tighter (more conservative) risk levels result in even less and smaller congestion issues and even fewer control actions but increases the curtailed energy.

\section{DISCUSSION}

This section discusses practical implementation aspects of the proposed risk-based NMS and potential improvements.

\footnotetext{
${ }^{1}$ Typical normal cyclic ratings used in the UK for underground cables range from $10-15 \%$ of the continuous rating.
} 


\section{A. Data Acquisition, Comms and Control}

The proposed approach requires different accurate parameters such as the network topology and set points (e.g., OLTCs, DG plants) as well as the streaming of demand and generation data. To reduce the potential errors in the data acquisition process (due to the measurement devices or noise in the communication channels) and potentially extend network observability a state estimator could be interfaced with the SCADA system [25]. In addition, if the errors can be statistically determined, the proposed risk-based approach could be further developed to incorporate them, similarly to the wind power changes.

Due to different factors controllable elements could in practice locally override optimal set points. If these new set points result in congestion or voltage issues, the proposed NMS will in the next control cycle make a new optimal decision based on the latest network state provided by the SCADA. In the event of loss of communications, fall back set points, particularly for the DG plants, could be implemented by which the network will not be comprised at any generation level [26].

It is also important to mention that the adoption of multiminute control cycles also allows reducing the need of high sampling rates, hence reducing the complexity and cost of a reliable communication infrastructure.

\section{B. Commercial Arrangements}

In practice, controllable DG plants agree with DNOs on specific commercial arrangements, i.e., the way that curtailment will be carried out. Although this has not been considered in this work, i.e., the optimal set points are found on technical grounds only, approaches such as Last In First Out (LIFO) or Pro Rata can be implemented [27], [28].

\section{Wind Power Transitions}

To provide a robust quantification of wind power transitions the historical assessment should be applied over a significant period. In this work, one-minute resolution data for one month was used. This analysis may be further improved by adopting a sliding window, i.e., updating the historic data periodically (e.g., each day or week) to consider the new wind power measurements.

\section{Risk Level}

The risk level is defined after assessing the performance of different values. Although in this work the study was limited to a week, the long term use of the NMS requires the risk level to be defined periodically, i.e., monthly or seasonally, to consider the corresponding changes in demand and wind generation.

\section{CONCLUSIONS}

This work demonstrates the effectiveness of a risk-based AC OPF as the decision engine of an advanced distribution Network Management System (NMS) aimed at maximizing the harvesting of wind energy whilst tackling congestion and voltage issues as well as the effects of wind uncertainty. The proposed approach allows adopting multi-minute control cycles, thus reducing the volume of control actions from
OLTCs and DG plants. The framework allows investigating in detail the benefits and impacts of adopting different control cycles, quantifying in particular compliance of voltages with the EN50160 standard, congestion levels, capacity factor of wind farms and, crucially, volume of control actions.

High-resolution operational aspects were implemented to optimally control OLTCs, DG power factor and DG curtailment (as last resort) in a way that the influence of control cycles and different risk levels on the overall performance of the NMS was investigated and compared against a simpler deterministic approach.

The application to a real-life UK MV network, considering high penetration of wind power capacity, revealed that, for multi-minute control cycles, the risk-based NMS always manages network constraints better than the deterministic approach given that the variability of wind power is considered at the decision time.

Tighter risk levels were found to improve the overall performance of the NMS optimization (in terms of congestion and voltages) even with longer control cycles. Although this also resulted in more curtailment, it could be of interest in cases where DNOs cannot implement very short control cycles due to practical issues such as the flexibility and response of controllable elements.

Ultimately, the proposed work can become an important tool for distribution network operators to understand the benefits and challenges of departing from deterministic NMS with multi-second-based control cycles to a risk-based one with multi-minute control cycles.

\section{REFERENCES}

[1] R. A. Walling, R. Saint, R. C. Dugan, J. Burke, and L. A. Kojovic, "Summary of Distributed Resources Impact on Power Delivery Systems," IEEE Transactions on Power Delivery, vol. 23 no. 3 pp. 1636 - 1644 July 2008.

[2] R. A. F. Currie, G. W. Ault, C. E. T. Foote, and J. R. McDonald, "Active power-flow management utilising operating margins for the increased connection of distributed generation," IET Proceedings Generation, Transmission \& Distribution, vol. 1, no. 1, pp. 197-202, Jan. 2007.

[3] T. Sansawatt, L. F. Ochoa, and G. P. Harrison, "Smart Decentralized Control of DG for Voltage and Thermal Constraint Management " IEEE Transactions on Power Systems, vol. 27, no. 3, pp. 1637 - 16452012.

[4] L. F. Ochoa, A. Keane, and G. P. Harrison, "Minimizing the reactive support for distributed generation: Enhanced passive operation and smart distribution networks," IEEE Trans. on Power Systems, vol. 26, no. 4, pp. 2134-2142, Nov. 2011.

[5] S. W. Alnaser and L. F. Ochoa, "Hybrid controller of energy storage and renewable DG for congestion management," in Proc. 2012 IEEE/PES General Meeting 2012 pp. 1-8.

[6] E. M. Davidson, S. McArthur, C. Yuen, and M. Larsson, "AuRA-NMS: Towards the delivery of smarter distribution networks through the application of multi-agent systems technology," in Proc. 2008 IEEE/PES General Meeting, pp. 6.

[7] "Grid 2020: Towards a Policy of Renewable and Distributed Energy Resources," The Ressnick Stustaiability Institute 2012.

[8] P. P. Varaiya, F. F. Wu, and J. W. Bialek, "Smart operation of smart grid: Risk-limiting dispatch," Proceedings of the IEEE, vol. 99, no. 1, pp. 4057, Jan. 2011.

[9] P. Daly, M. Power, A. Keane, and D. Flynn, "Operational security at high penetrations of stochastic, non-synchronous generation," in Proc. 2013 International Universities Power Engineering Conference (UPEC).

[10]T. Xu, N. S. Wade, E. M. Davidson, P. C. Taylor, S. D. J. McArthur, and W. G. Garlick., "Case-Based Reasoning for Coordinated Voltage Control on Distribution Networks," Electric Power Systems Research vol. 81, no. 12, pp. 2088-2098, December 20112011.

[11]M. J. Dolan, E.M.Davidson, G. W. Ault, K.R.W.Bell, and S. D. J. McArthur, " Distribution Power Flow Management Utilizing an Online 
Constraint Programming Method," IEEE Transactions on Smart Grid, vol. 4, no. 2, pp. 798 - 805, 2013.

[12]M. J. Dolan, E. M. Davidson, I. Kockar, G. W. Ault, McArthur, and S.D.J., "Distribution Power Flow Management Utilizing an Online Optimal Power Flow Technique " IEEE Transactions on Power Systems, vol. 27, no. 2, pp. 790-799, May 2012.

[13]F. Pilo, G. Pisano, and G. G. Soma, "Optimal Coordination of Energy Resources With a Two-Stage Online Active Management " IEEE Transactions on Industrial Electronics, vol. 58, no. 10, pp. 4526 - 4537 Oct. 2011.

[14]H. Zechun and L. Fu-Rong, "Cost-benefit analyses of active distribution network management, part I : Annual benefit analysis," IEEE Transactions on Smart Grid,, vol. 3, no. 3, pp. 1067-1074, 2012.

[15]M. J. Dolan, E. M. Davidson, I. Kockar, G. W. Ault, and S. D. J. McArthur, "Reducing Distributed Generator Curtailment Through Active Power Flow Management," IEEE Transactions on Smart Grid, vol. 5, no. 1, pp. 149 - 1572014.

[16]UK Department of Trade and Industry, The electricity supply, quality and continuity regulations 2002, Sep. 2002.

[17]L. F. Ochoa, C. Dent, and G. P. Harrison, "Distribution network capacity assessment: Variable DG and active networks," IEEE Trans. on Power Systems, vol. 25, no. 1, pp. 87-95, Feb. 2010.

[18]R. Dugan and T. E. McDermott, "An open source platform for collaborating on smart grid research," in IEEE/PES General Meeting 2011, 2011, pp. 1 - 7

[19]J. Bisschop and M. Roelofs, "AIMMS - The user's guide," Paragon Decision Technology, 2006.

[20]A. Shapiro, D. Dentcheva, and A. P. Ruszczyńsk, Lectures on stochastic programming : modeling and theor: Philadelphia : Society for Industrial and Applied Mathematics : Mathematical Programming Society, 2009.

[21]S. Engelhardt, I. Erlich, C. Feltes, J. Kretschmann, and F. Shewarega, "Reactive power capability of wind turbines based on doubly fed induction generators," IEEE Trans. on Smart Grid, vol. 26, no. 1, pp. 364372, Mar. 2011.

[22]G. Giebel, R. Brownsword, G. Kariniotakis, M. Denhard, and C. Draxl. The State of the Art in Short-Term Prediction of Wind PowerA Literature Overview A Literature Overview, 2nd Edition. [Online]. Available: http://www.anemos-plus.eu/

[23]S. Skogestad, "Simple analytic rules for model reduction and PID controller tuning " Journal of Process Control, vol. 13, pp. 291-309, 2003.
[24]National Grid Electricity Transmission, The Grid Code Aug. 2012.

[25]R.Singh, B. C. Pal, and R. A. Jabr, "Choice of estimator for distribution system state estimation," IET Proceedings Generation, Transmission \& Distribution, vol. 3, no. 7, pp. 666 - 6782009.

[26]R. A. F. Currie, G. W. Ault, D. F. MacLeman, and R. W. Fordyce. The Orkney RPZ: Facilitating Increased Connection of Renewable Generation through Active Network Management. [Online]. Available: http://www.ssepd.co.uk/uploadedFiles/SSEPD_Microsites/Orkney_smart grid/Controls/Resources/TheOrkneyRegisteredPowerZone.pdf

[27]R. A. F. Currie, B. O’Neill, C. Foote, A. Gooding, R. Ferris, and J. Douglas, "Commercial arrangements to facilitate active network management," in CIRED 21st International Conference on Electricity Distribution, 2011.

[28]L. Kane, G. Ault, and S. Gill, "An assessment of principles of access for wind generation curtailment in active network management schemes," in 22nd International Conference on Electricity Distribution (CIRED), 2013, pp. 5.

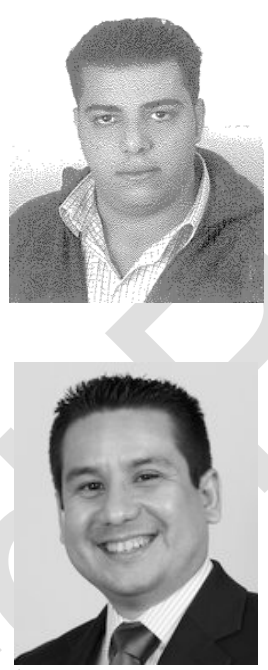

Sahban W. Alnaser (S’11) received the B.Sc. and M.Sc. degrees in Electrical Engineering from the University of Jordan, Jordan, in 2005 and 2008, respectively. $\mathrm{He}$ is currently pursuing the Ph.D. degree at The University of Manchester, Manchester, U.K. From 2005 to 2011 he was with the Electricity Distribution Company (EDCO) in Jordan as head of power system studies section. His research interests include integration of distributed energy resources.

Luis F. Ochoa (S'01, M'07, SM'12) received the B.Eng. degree from UNI, Lima, Peru, in 2000 and the M.Sc. and Ph.D. degrees from UNESP, Ilha Solteira, Brazil, in 2003 and 2006, respectively. He is a Lecturer in Smart Distribution Networks at The University of Manchester, UK. His current research interests include network integration of distributed energy resources and future low-carbon distribution networks. 